\title{
The role of inflammatory mediators in the pathogenesis of nasal polyposis: Literature review
}

\author{
Roxana Duda \\ "Carol Davila" University of Medicine and Pharmacy, Bucharest, Romania
}

\begin{abstract}
Nasal polyposis represents a late stage of long-lasting inflammation of the sinonasal mucosa, characterized by increased inflammatory cells infiltration and anomalous tissue remodelling. A wide range of chemical mediators such as cytokines, chemokines, cell adhesion molecules are involved in the pathomechanism of nasal polyposis, but their significance has not been completely clarified.

Eosinophils are the dominant inflammatory cell population in nasal polyposis and are thought to be the central effector cells responsible for the onset and maintenance of the inflammatory process. Persistent inflammation of the sinonasal mucosa can lead to structural changes, such as epithelial damage, thickened basement membrane, stromal edema, formation of pseudocysts. This review summarizes prior and current knowledge regarding the involvement of the inflammatory process in the pathogenesis of nasal polyposis.
\end{abstract}

KEYWORDS: nasal polyposis, eosinophils, cytokines, chemokines, cell adhesion molecules.

\section{INTRODUCTION}

Nasal polyposis is a complex inflammatory disease with a predominance of eosinophils infiltration, regulated by $\mathrm{T}$ lymphocyte subsets and represents a late stage of long-lasting inflammation of the sinonasal mucosa ${ }^{1,2}$. The increased inflammatory cells infiltration and persistent inflammation can lead to structural changes of the respiratory epithelium, resulting a unique remodelling process ${ }^{3}$.

Various hypotheses regarding the underlying mechanism of nasal polyposis have been put forward, including chronic infection, nasal epithelial disruptions, allergies, inflammatory-bioelectric theory, aerodynamic factors, immune system malfunctions. Despite remarkable scientific progress, the pathogenesis of nasal polyposis has not yet been fully elucidated ${ }^{4}$.

The chronic inflammatory specific feature, tendency to recurrence, troublesome symptoms, lack of satisfying treatment suggest that this entity remains a serious health problem worldwide, with significant socioeconomic consequences.

Research made in the last decade has documented an impairment in the regulation of a wide range of inflammatory markers and cell types in patients with nasal polyposis ${ }^{4,5}$.
Histologically, nasal polyposis is typically characterized by epithelial damage, thickened basement membrane, intense edematous stroma with albumin deposition, formation of pseudocysts, accompanied by the infiltration of inflammatory cells mainly consisting of eosinophils, but also mast cells, macrophages, lymphocytes and neutrophils ${ }^{6}$. Eosinophils are the dominant inflammatory cell population in nasal polyposis and are thought to be the central effector cells responsible for the onset and maintenance of the inflammatory process $^{7}$. Mechanisms underlying the activation, recruitment and survival of eosinophils involve a complex interaction of multiple inflammatory cells and pathways. Increased synthesis and expression of an affluence of cytokines ((IL-1, IL-3, IL-5, IL-6, IFN $\alpha$ ) and chemokines (IL-8, eotaxin, RANTES) play a crucial role in eosinophils survival and activation, mediating these processes ${ }^{7,8}$.

Another important class of inflammatory mediators is the adhesion molecules (ICAM-1, VCAM-1), which are responsible for the migration of inflammatory cells to the damaged tissue. Recent studies offer important and needful information on the pattern of local inflammation and its related pathologic mecha$n^{n i s m}{ }^{8}$. The following material summarizes prior and current knowledge concerning the involvement of inflammatory cells in nasal polyposis. 


\section{INFLUENCE OF CHEMICAL MEDIATORS ON EOSINOPHILIC INFLAMMATION}

In nasal polyposis, for reasons not fully clarified, there is an astonishing influx of inflammatory cells, predominantly eosinophils, into the stroma, which activate a positive feedback loop by recruiting additional eosinophils. Eosinophil tissue accumulation stimulates other inflammatory cells inducing an amplification of the inflammatory process, thus maintaining the vicious cycle .

In an attempt to better understand the inflammatory process of nasal polyposis, it is helpful to define more clearly the mechanisms underlying the activation, migration and maintenance of eosinophils ${ }^{10}$. Eosinophils play a dominant role in the development of nasal polyposis, being the main infiltrating inflammatory cells. Eosinophils are recruited from the peripheral blood circulation by chemotactic factors and activated into inflamed tissues, in response to inflammatory-derived mediators ${ }^{10,11}$. At the site of inflammation, they perform their end-phase effector function through differential secretion of proinflammatory mediators and cytotoxic proteins. They produce a broad array of highly toxic preformed granule proteins (major basic protein-MBP, eosinophil cationic protein-ECP, eosinophil peroxidase-EPO), different cytokines (IL-1, IL-3, IL-4, IL-5, IL-10, IL-12, GM-CSF, INF $\alpha, T N F \alpha$ ), chemokines (IL-8, eotaxin, RANTES), with different target activities. These mediators have pro-inflammatory effects in the upregulation of adhesion molecules (ICAM-1, VCAM-1), modulation of cellular trafficking and induction tissue damage ${ }^{11,12}$.

\section{CYTOKINES}

Proinflammatory cytokines represent key signalling molecules, involved in processes such as chemotaxis, control of cellular proliferation, cell activation, differentiations and function of many cells participating in the inflammatory process.

Many studies that have focused on the cytokines responsible for eosinophils function have shown that these include interleukins such as IL-1, IL-3, IL-4, IL-5, IL-10, GM-CSF, TNF $\alpha$, even if each of them is implicated in a different manner ${ }^{12}$. Of great importance are IL-3, IL-5 and GM-CSF that induce eosinophilic effector function and survival of eosinophils and also stimulate eosinophils to express a variety of receptors for cytokines $^{12,13}$. IL-3 is a cytokine which promoting the development and functional activity of various haematopoietic cell types, including eosinophils.

GM-CSF is an important haematopoietic growth factor which stimulates proliferation, activation and differentiation of macrophages, neutrophils and eosinophils ${ }^{14}$.
IL-5 is a cytokine that stimulates growth and differentiation of the eosinophil lineage in the bone marrow, mediates the activation and selective migration of eosinophils from the peripheral circulation into the tissue, increases the vitality of eosinophils by impeding their apoptosis $^{14,15}$. IL-3, IL-5 and GM-CSF share similar functions when they act on the same cells. In vitro studies have assessed the ability of IL-3, IL-5 and GM-CSF to improve eosinophil survival. One of their most important effects is to prolong eosinophil life span by deferring the onset of apoptosis ${ }^{16,17}$. Of these, IL-5 has been recognized as the most specific cytokine to eosinophils and represents the main key driver regulator of eosinopoiesis. Interleukin 5 is produced by Th 2 cells, although it can also be synthesized and released by eosinophils, stirring an autocrine inflammatory process that is able to maintain the persistent eosinophilia. The role of IL-5 in the pathogenesis of nasal polyposis is supported by several lines of experimental evidence. Elevated levels of IL-5 found in nasal polyposis highlight the importance of this cytokine in the pathogenesis of this disease ${ }^{17}$. Fan et al. in their study suggest that IL-5 secretion induced by Th 2 cell and autosecretion of IL-5 from activated eosinophils may be the cause reasons for the onset and maintenance of eosinophil inflammation in the nasal polyp tissue ${ }^{18}$. Another study led by Hirschberg et al. revealed that IL-5 is a pivotal factor involved in the eosinophil recruitment and activation ${ }^{19}$.

The production and activity of eosinophils are significantly directed by IL-5 via the IL-5 receptor ( $\alpha$ chain), specifically expressed on their surface and precursors, pointing out its role in the inflammatory pro$\operatorname{cess}^{20}$.

Evaluation of the apoptotic behaviour of eosinophils through in vitro studies demonstrates that IL-5 decreases the rate of eosinophil programmed cell death ${ }^{19,20}$. According to Jankowski et al., eosinophils, even at lower levels, play an important role in the development of polyps ${ }^{21}$. Ex vivo studies have shown that the eosinophil life span was reduced by incubation with anti-IL-5 monoclonal antibodies, indicating the significant role of IL-5 in the regulation of eosinophil survival.

Current research studies have provided evidence suggesting that staphylococcal super antigens can induce the synthesis of IL-5 and promote eosinophil activation.

Aside from its effects on eosinophils, IL-5 enhances the expression of the adhesion molecules VCAM-1and ICAM-1. In addition to activating eosinophils, IL-3 also downregulates the chemokine receptor CCR3 on the surface of eosinophils. The two cytokines IL-3 and IL-5 work in tandem to induce eosinophil differentiation from progenitor cells ${ }^{22}$.

Dellacono et al. were able to establish the existence of a positive correlation between the GM-CSF-mRA levels and the number of eosinophils in nasal polyps ${ }^{23}$. 
IL-5 in combination with IL-1 $\beta$ and TNF $\alpha$ increase transendothelial migration of eosinophils. Although it has been demonstrated that TNF $\alpha$ and IL-1 enhance eosinophil life span in vitro, it has also been investigated their ability to modulate the expression of several cell adhesion molecules on endothelial cells. IL-1 $\beta$ has been proposed to play a crucial role in the pathogenesis of nasal polyps ${ }^{24,25}$. Through its action this cytokine, it can increase transendothelial migration of eosinophils. In the study by Saji et al., investigators noted that eosinophil infiltration was induced by fibroblasts through the action of IL-1 $\beta^{26}$.

The concentrations of some other proinflammatory cytokines, such as IL-6, IL-9, IL-13, are higher in patients with nasal polyposis. In vitro studies developed over the last years have pointed that IL-13 promotes the chemotaxis and prolongs the survival of eosinophils.

Despite the fact that there is a large array of cytokines that affect the activity of eosinophils, these findings collectively suggest that IL-5 is one of the most influential inflammatory mediators for eosinophils activity ${ }^{27}$.

\section{CHEMOKINES}

As cytokines, chemokines became the target of intense debate regarding their importance in coordinating the function and trafficking of eosinophils. Increased expression and production of chemokines found in nasal polyposis developed an intense interest regarding their mechanism of action and binding. Various functions have been assigned to chemokines, including proinflammatory activities such as chemotaxis, degranulation, functions which are mediated by receptors. Signals elicited through chemokines receptors play an important role in the inflammatory process of nasal polyposis. Experimental observations suggest that there is an interdependence between the number of eosinophils and the tissue concentration of the individual chemokines ${ }^{28,29}$. Eosinophils generate a large number of chemokines, including MIP, MCP RANTES, eotaxin, which represent major regulators of local inflammatory response ${ }^{30}$. Eotaxin and RANTES are two factors of particular significance with chemotactic and activating effects in eosinophils ${ }^{31}$. Eotaxin is a potent stimulus for eosinophils, inducing eosinophil migration and accumulation via high affinity to the chemokine receptor 3 (CCR3) ${ }^{32}$.

Recent research indicates that all members of the Eotaxin family $(1,2,3)$ play key roles in the inflammatory process of nasal polyposis. Significantly increased amounts of Eotaxin 1, Eotaxin 2, Eotaxin 3 were present in the polyp tissue, when compared with the control tissue. Various authors have demonstrated through in vitro and in vivo experiments, the specific chemotactic potency of all eotaxins. Eotaxin is a specific chemoattractant for eosinophils. It is produced by cytokine-stimulated epithelial and endothelial cells as well as IL-3-stimulated eosinophils ${ }^{32,33}$.

Eotaxin has been shown to induce eosinophil migration in vivo. Multiple findings suggest that nasal polyps are a rich source of eotaxin ${ }^{34}$. Eotaxin 1 , along with eosinophil survival factor IL-5, was found to be implicated in eosinophil recruitment in nasal polyposis. Eotaxin may favour eosinophil accumulation to the nasal mucosa endothelial cells through its effect on the selective expression of endothelial adhesion molecules. Hence, eotaxin has multifaceted effects on eosinophils and is likely to play a key role in determining the increase of tissue eosinophilia ${ }^{34,35}$.

In addition to eotaxin, another important chemokine strongly related to nasal polyposis is RANTES (Regulated on Activation, Normal T Cell Expressed and Secreted). RANTES is a chemokine with chemotactic activity mainly on eosinophils and T lymphocytes. High levels of RANTES lead to increased tissue eosinophilia and induce the recruitment and activation of eosinophils. Previous studies have demonstrated that RANTES has been shown to be a potent mediator of eosinophil chemotaxis ${ }^{36}$. RANTES is an important recruiter and activator of eosinophils and its relationship to nasal polyposis has been analyzed in different manners ${ }^{37,38}$. Comparing polyps with control specimens, Lane et al. noted higher expression of RANTES in nasal polyposis ${ }^{39}$. Apart from high amounts of RANTES found in nasal polyps specimens, Saji et al. have also demonstrated that nasal polyp fibroblasts play a major role in the mechanism of eosinophil recruitment through RANTES production ${ }^{40}$.

Another important chemokine implicated in nasal polyposis pathogenesis is IL-8. This chemokine represents a potent chemoattractant for neutrophils, but also has chemotactic effect on eosinophils, attracting them to the site of inflammation ${ }^{41}$. Data regarding its role in the development of nasal polyposis report that IL-8 is an important chemokine with chemotactic activity for eosinophils. According to most recent studies, IL-8 is an important chemokine in the pathogenesis of nasal polyposis, as demonstrated by elevated levels detected in tissue samples. It has been demonstrated that IL-8 delivers signals that lead to selective influx of eosinophils ${ }^{41,42}$

\section{CELL ADHESION MOLECULES}

An assessment of the unique and complex mechanism responsible for mobilization of eosinophils into the airways lumen has established that this includes also a range of both adhesion molecules expressed on 
eosinophils and endothelial cells, such as ICAM-1, VCAM-1, selectins (E-selectin, P-selectin), integrins ${ }^{43}$.

It has been suggested that adhesion molecules play a significant role in the pathogenesis of nasal polyposis, although this class of inflammatory mediators needs to be better studied ${ }^{44}$. In biopsies taken from patients with nasal polyposis, increased levels of ICAM1, VCAM-1, E- selectin have been noticed ${ }^{45}$.

In vitro studies have found that VCAM-1 is an important and relatively selective chemoattractant for eosinophils, mediating recruitment, sequestration and transendothelial migration of these cells ${ }^{46}$. The research of several groups has proved that increased levels of VCAM-1 detected in patients with nasal polyposis were correlated with the presence of eosinophils, leading to the assumption that eosinophil recruitment is, in part, mediated by VCAM- $1{ }^{47}$.

In addition to increased levels of VCAM-1, Beck et al in their findings have found a positive correlation between VCAM-1 and the number of eosinophils ${ }^{48}$.

Moreover, ICAM-1 has been revealed to be expressed in vascular endothelial cells and implicated in the migration of eosinophils from blood vessels to tissues and eosinophil infiltration. Thus, ICAM-1 plays a major role in promoting proinflammatory intercellular response; its role in nasal polyposis has not been elucidated despite its elevated levels of expression ${ }^{49}$.

Aside from VCAM-1, another adhesion molecule able to stimulate the recruitment of eosinophils in nasal polyposis is P-selectin ${ }^{50}$.

\section{CONCLUSIONS}

Although nasal polyposis is considered a multifactorial disease with still debated pathogenesis, numerous studies have demonstrated that eosinophil infiltration, regulated by $\mathrm{T}$ lymphocyte subsets, is the main key feature. Nasal polyposis remains a major health problem worldwide, which creates a serious financial and psychosocial impact.

A growing body of evidence demonstrated that nasal polyposis is a complex interplay of numerous cytokines, chemokines, adhesion cells and receptors. Investigation of individual inflammatory mediators is essential for enhancing the understanding of their precise contribution to the initiation and maintenance of chronic inflammation in this disease.

The present studies definitely provide significant information to further expand the research in this field.

Additional research is needed to further explain and define the complexity of the mechanisms responsible for chronic inflammatory response in nasal polyposis. Given the inflammatory condition of nasal polyposis, elucidation of the underlying inflammatory pat- terns is indispensable for developing new therapeutic strategies.

\section{REFERENCES}

1. Alexiou A., Sourtzi P., Dimakopoulou K., Manolis E., Velonakis E. - Nasal polyps: heredity, allergies, and environmental and occupational exposure. J Otolaryngol Head Neck Surg., 2011;40:58-63.

2. Van Bruaene N., Perez Novo C., Deruyck N., Holtappels G., Van Cauwenberge P., Bachert C. - Inflammation and remodeling patterns in early-stage chronic rhinosinusitis. Clin Exp Allergy, 2012;42:883-890.

3. Van Bruaene N., Bachert C. - Tissue remodeling in chronic rhinosinusitis. Curr Opin Allergy Clin Immunol., 2011;11:8-11.

4. Anand V.K. - Epidemiology and economic impact of rhinosinusitis. Ann Otol Rhinol Laryngol. Suppl., 2004;193:3-5.

5. Drake-Lee A. - The pathogenesis of nasal polyps. In: Settipane G.A., Lund V.J., Tos M. - Nasal polyps: epidemiology, pathogenesis and treatment. Providence, Rhode Island: Ocean Side Publications, 1997;p.57-64.

6. Kim J.W., Hong S.L., Kim Y.K., Lee C.H., Min Y.G., Rhee C.S. - Histological and immunological features of non-eosinophilic nasal polyps. Otolaryngol Head Neck Surg., 2007;137(6):925-930.

7. Simon H.U., Yousefi S., Schranz C., Schapowal A., Bachert C., Blaser K. Direct demonstration of delayed eosinophil apoptosis as a mechanism causing tissue eosinophilia. J Immunol., 1997;158(8):3902-3908.

8. Kim Y.M., Munoz A., Hwanq P.H., Nadeau K.C. - Migration of regulatory T cells toward airway epithelial cells is impaired in chronic rhinosinusitis with nasal polyposis. Clin Immunol., 2010;137(1):111-121. doi: 10.1016/j. clim.2010.05.013. Epub 2010 Jul 2.

9. Kato A., Schleimer R.P. - Beyond inflammation: airway epithelial cells are at the interface of innate and adaptive immunity. Curr Opin Immunol., 2007;19(6):711-720.

10. Di Lorenzo G., Drago A., Esposito Pellitteri M., Candore G., Colombo A., Gervasi F., Pacor M.L., Purello D’Ambrosio F., Caruso C. - Measurement of inflammatory mediators of mast cells and eosinophils in native nasal lavage fluid in nasal polyposis. Int Arch Allergy Immunol., 2001;125(2):164-175.

11. Erbek S.S., Yurtcu E., Erbek S., Atac F.B., Sahin F.I., Cakmak O. Proinflammatory cytokine single nucleotide polymorphisms in nasal polyposis. Arch Otolaryngol Head Neck Surg., 2007;133(7):705-709.

12. Van Zele T., Claeys S., Gevaert P., Holtappels G., Van Cauwenberge P., Bachert C. - Differentiations of chronic sinus diseases by measurement of inflammatory mediators. Allergy, 2006;61(11):1280-1289.

13. Hamilos D.L., Leung D.Y.M., Wood R., Cunningham L., Bean D.K., Yasruel S., Schotman E., Hamid Q. - Evidence for distinct cytokine expression in allergic versus nonallergic chronic sinusitis. J Allergy Clin Immunol., 1995;96:537-544.

14. Otto B.A., Wenzel S.E. - The role of cytokines in chronic rhinosinusitis with nasal polyps. Curr Opin Otolaryngol Head Neck Surg., 2008;16(3):270-4. doi: 10.1097/ moo.0b013e3282fb2885.

15. Gevaert P., Lang-Loidolt D., Lackner A., Stammberger H., Staudinger H., Van Zele T., Holtappels G., Tavernier J., van Cauwenberge P., Bachert C. - Nasal IL-5 levels determine the response to anti-IL-5 treatment in patients with nasal polyps. J Allergy Clin Immunol., 2006;118(5):1133-1141. Epub 2006 Sep 26.

16. de Borja Callejas F., Picado C., Martínez-Antón A., Alobid I., Pujols L., Valero A., Roca-Ferrer J., Mullol J. - Differential expression of remodeling markers by tissue structure in nasal polyposis. Am J Rhinol Allergy, 2013;27(3):e69-74. doi: 10.2500/ajra.2013.27.3908. 
17. Hamilos D.L., Leung D.Y.M., Huston D.P., Kamil A., Wood R., Hamid Q. GM-CSF, IL-5 and RANTES immunoreactivity and mRNA expression in chronic hyperplastic sinusitis with nasal polyposis (NP). Clin Exp Allergy, 1998;28:1145-1152.

18. Peri囚 A., Vijvodic D., Vukomanovic-Durdevic B., Baletic N. - Inflammatory mediators in allergic rhinitis and nasal polyposis. Arh Hig Rada Tokiskol., 2011;62(4):341-348. doi: 10.2478/10004-1254-62-2011-2120.

19. Hirschberg A., Jokuti A., Darvas Z., Almay K., Repassy G., Falus A. - The pathogenesis of nasal polyposis by immunoglobulin $\mathrm{E}$ and interleukin-5 is completed by transforming growth factor-betal. Laryngoscope, 2003;113(1):120-124.

20. Gavaert P., Van Bruaene N., Cattaert T., Van Steen K., Van Zele T., Acke F., De Ruyck N., Blomme K., Sousa Ana R., Marshall Richard P., Bachert C. - Mepolizumab, a humanized anti-IL-5 mAb, as a treatment option for severe nasal polyposis. The Journal of Allergy and Clinical Immunology, 2011;128(5):989-995.e8.

21. Jankowski R., Moneret-Vautrin D.A., Goetz R., Wayoff M. - Incidence of medico-surgical treatment for nasal polyps on the development of associated asthma. Rhinology, 1992;30(4):249-258.

22. Clutterbuck E.J., Hirst E.M., Sanderson C.J. - Human interleukin-5 (IL-5) regulates the production of eosinophils in human bone marrow cultures: comparison and interaction with IL-1, IL-3, IL-6, and GMCSF. Blood, 1989;73(6):1504-1512.

23. Dellacono F.R., Eisma R., Lafreniere D., Leonard G., Kreutzer D. Interferon gamma expression in human nasal polyps. Laryngoscope, $1997 ; 107(5): 626-630$.

24. Mfuna Endam L., Cormier C., Bosse Y., Filali-Mouhim A., Desrosiers M. Association of IL1A, IL1B, and TNF gene polymorphisms with chronic rhinosinusitis with and without nasal polyposis: A replication study. Arch Otolaryngol Head Neck Surg., 2010;136:187-192. doi: 10.1001/archoto.2009.219.

25. Kato A., Peters A., Suh L., Carter R., Harris K.E., Chandra R., Conley D., Grammer L.C., Kern R., Schleimer R.P. - Evidence of a role for B cell-activating factor of the TNF family in the pathogenesis of chronic rhinosinusitis with nasal polyps. J Allergy Clin Immunol., 2008;121(6):1385-1392, 1392.e1-2. doi: 10.1016/jaci.2008.03.002. Epub 2008 Apr 14.

26. Saji F., Nonaka M., Pawankar R. - Expression of RANTES by IL-1 beta and TNF-alpha stimulated nasal polyp fibroblasts. Auris Nasus Larynx, 2000;27:247-252.

27. Rosenberg H.F., Dyer K.D., Foster P.S. - Eosinophils: changing perspectives in health and disease. Nat Rev Immunol., 2013;13(1):9-22. doi: 10.1038/ hri3341. Epub 2012 Nov 16.

28. Gounni Abdelilah S., Wellemans V., Agouli M., Guenounou M., Hamid Q., Beck L.A., Lamkhioued B. - Increased expression of Th2-associated chemokines in bullous pemphigoid disease: Role of eosinophils in the production and release of these chemokines. Clin Immunol., 2006;120:220231 .

29. Liu L.Y., Bates M.E., Jarjour N.N., Busse W.W., Bertics P.J., Kelly E.A. Generation of Th1 and Th2 chemokines by human eosinophils: evidence for a critical role of TNF-alpha. J Immunol., 2007;179(7):4840-4848.

30. Bachert C., Gevaert P., Holtappels G., Cuvelier C., van Cauwenberge P. Nasal polyposis: from cytokines to growth. Am J Rhinol., 2000;14(5):279290.

31. Patadia M., Dixon J., Conley D., Chandra R., Peters A., Suh L.A., Kato A., Carter R., Harris K., Grammer L., Kern R., Schleimer R. - Evaluation of the presence of B-cell attractant chemokines in chronic rhinosinusitis. Am J Rhinol Allergy, 2010;24(1):11-6. doi: 10.2500/ajra.2010.24.3386.

32. Bartels J., Maune S., Meyer J.E., Kulke R., Schleter C., Rowert J., Christophers E., Schroeder J.M. - Increased eotaxin mRNA expression in non atopic and atopic nasal polyps: comparison to RANTES and MCP3 expression. Rhinology, 1997;35(4):171-174.

33. Yao T., Kojima Y., Koyanagi A., Yokoi H., Saito T., Kawano K., Furukawa M., Kusunoki T., Ikeda K. - Eotaxin-1, -2, and -3 immunoreactivity and protein concentration in the nasal polyps of eosinophilic chronic rhinosinusitis patients. Laryngoscope, 2009;119(6):1053-1059. doi: 10.1002/lary.20191.

34. Palframan R.T., Collins P.D., Williams T.J., Rankin S.M. - Eotaxin induces a rapid release of eosinophils and their progenitors from the bone marrow. Blood, 1998;91 (7):2240-2248.

35. Cavallari F.E., Valera F.C.P., Gallego A.J. et al. - Expression of RANTES, eotaxin-2, ICAM-1, LFA-1 and CCR-3 in chronic rhinosinusitis patients with nasal polyposis. Acta Cirurgica Brasileira, 2012;27(9):645-649.

36. Allen J.S., Eisma R., LaFreniere D., Leonard G., Kreutzer D. Characterization of the eosinophil chemokine RANTES in nasal polyps. Ann Otol Rhinol Laryngol., 1998;107(5pt1):416-420.

37. Newton J.R., Ah-See K.W. - A review of nasal polyposis. Ther Clin Risk Manag., 2008;4(2):507-512.

38. Shin S.H., Park J.Y., Jeon C.H., Choi J.K., Lee S.H. - Quantitative analysis of eotaxin and RANTES messenger RNA in nasal polyps: association of tissue and nasal eosinophils. Laryngoscope, 2000;110(8):1353-1357.

39. Lane A.P., Truong-Tran Q.A., Schleimer R.P. - Altered expression of genes associated with innate immunity and inflammation in recalcitrant rhinosinusitis with polyps. Am J Rhinol., 2006;20(2):138-144.

40. Meyer J.E., Bartels J., Gorogh T., Sticherling M., Rudack C., Ross D.A., et al. - The role of RANTES in nasal polyposis. Am J Rhinol., 2005;19(1):15-20.

41. Ohkubo K., Masakazu I., Pawankar R., Gotoh M., Yagi T., Okuda M. Mechanisms of IL-6, IL-8 and GM-CSF release in nasal secretions of allergic patients after nasal challenge. Rhinology, 1998;36:156-161.

42. Allen J.S., Eisma R., Leonard G., Lafreniere D., Kreutzer D. - Interleukin-8 expression in human nasal polyps. Otolaryngol Head Neck Surg., 1997;117(5):535-541.

43. Corsi M.M., Pagani D., Dogliotti G., Perona F., Sambataro G., Pignataro L. - Protein biochip array of adhesion molecule expression in peripheral blood of patients with nasal polyposis. Int J Biol Markers., 2008;23(2):115120 .

44. Gonzalo J.A., Lloyd C.M., Kremer L., et al. - Eosinophil recruitment to the lung in a murine model of allergic inflammation. The role of $\mathrm{T}$ cells, chemokines, and adhesion receptors. J Clin Invest., 1996;98(10):2332-2345.

45. Olejniczak I., Kobos J., Gryczy凶ski M., Durko M., Pietruszewska W. Expression of adhesion molecule ICAM-1 in patients with nasal polyps. Otolaryngol Pol., 2007;61(4):607-611. doi: 10.1016/S0030 6657(07)70495-X

46. Sriramarao P., Broide D.H. - Differential regulation of eosinophil adhesion under conditions of flow in vivo. Ann NY Acad Sci., 1996;796:218-225.

47. Eweiss A., Dogheim Y., Hassab M., Tayel H., Hammad Z. - VCAM-1 and eosinophilia in diffuse sino-nasal polyps. Eur Arch Otorhinolaryngol., 2009;266:377-383. doi: 10.1007/s00405-008-0762-1.

48. Gosset P., Tillie-Leblond I., Janin A., Marguette C.H., Copin M.C., Wallaert B., Tonnel A.B. -Expression of E-selectin, ICAM-1 and VCAM-1 on bronchial biopsies from allergic and non-allergic asthmatic patients. Int Arch Allergy Immunol., 1995;106(1):69-77.

49. Papon J.F., Coste A., Gendron M.C., Cordonnier C., Wingerstmann L., Peynegre R., Escudier E. - HLA-DR and ICAM-1 expression and modulation in epithelial cells from nasal polyps. Laryngoscope, 2002;112(11):20672075 .

50. Symon F.A., Walsh G.M., Watson S.R., Wardlaw A.J. - Eosinophil adhesion to nasal polyp endothelium is P-selectin-dependent. J Exp Med., $1994 ; 180(1): 371-376$. 